\title{
REVIEW ON HYDROTROPY: A POTENTIAL APPROACH FOR THE SOLUBILITY ENHANCEMENT OF POORLY SOLUBLE DRUG
}

\author{
JYOTI JOSHI*, NIDHI NAINWAL, VIKAS ANAND SAHARAN
}

Department of Pharmaceutical Sciences, School of Pharmaceutical Sciences and Technology, Sardar Bhagwan Singh Post Graduate Institute of Biomedical Sciences and Research, Balawala, Dehradun, Uttarakhand, India. Email: joshijyoti386@gmail.com

Received: 07 July 2019; Revised and Accepted: 10 August 2019

\begin{abstract}
Solubility is one of the significant parameters to accomplish wanted centralization of medication in foundational flow for pharmacological reaction to appear. Medication adequacy can be seriously constrained by poor watery dissolvability, and a few medications additionally show reactions because of their poor solvency. There are numerous strategies which are utilized to upgrade the fluid solvency. The capacity to increment watery solvency would thus be able to be a profitable guide to expanding proficiency as well as lessening reactions for specific medications. The ability to augment watery dissolvability would subsequently have the option to be a beneficial manual for extending capability just as diminishing responses for explicit medications. Therapeutic adequacy of a medication relies on the bioavailability and at last on the solvency of medication particles. Solvency is one of the significant parameters to accomplish wanted convergence of medication in fundamental course for pharmacological reaction to appear. Because of cutting edge inquire about and advancement, there are assortments of new medications and their subordinates are accessible. In any case, over $40 \%$ of lipophilic medication up-and-comers neglect to achieve showcase because of poor bioavailability, despite the fact that these medications may display potential pharmacodynamic exercises. The lipophilic medication that achieves market requires a high portion to accomplish appropriate pharmacological activity. Hydrotropy is one of the solvency improvement methods which upgrade dissolvability to numerous folds with utilization of hydrotropes such as sodium benzoate, sodium citrate, urea, niacinamide, and so forth and have numerous focal points as, it does not require substance alteration of hydrophobic medications, utilization of natural solvents, or readiness of emulsion framework and so forth.
\end{abstract}

Keywords: Hydrotropes, Solubility enhancement, Hydrophobic drugs, Bioavailability, Mixed hydrotropy.

(C) 2019 The Authors. Published by Innovare Academic Sciences Pvt Ltd. This is an open access article under the CC BY license (http://creativecommons. org/licenses/by/4. 0/) DOI: http://dx.doi.org/10.22159/ajpcr.2019.v12i10.34811

\section{INTRODUCTION}

Solubility is characterized in quantitative terms as the grouping of solute [1] in an immersed arrangement at a specific temperature, and in a subjective manner, it tends to be characterized as the unconstrained association of at least two substances to frame a homogeneous atomic scattering. The dissolvability of a compound relies on the physical and concoction properties of the solute and the dissolvable just as on such factors as temperature, weight, the $\mathrm{pH}$ of the arrangement, and so on. Thermodynamic balance solvency is accomplished when the by and large most minimal vitality condition of the framework is achieved [2]. The United States Pharmacopeia (USP) depicts the dissolvability of medications as parts of dissolvable required for one section solute. Dissolvability is additionally quantitatively communicated regarding molality, molarity, and rate.

In the ongoing years, present-day tranquilize disclosure endeavors have been delivering increasingly more lipophilic medication competitors, and as indicated by some perception over $70 \%$ of new medication atoms display poor water solubility [3]. The utilization of various polymer to empowering solvency is regular practice to handling dissolvability.

It is notable that medication viability can be seriously constrained by poor fluid dissolvability. It is additionally realized that the symptoms of certain medications are the consequence of their poor dissolvability. With the goal that the capacity to increment watery dissolvability would thus be able to be a profitable guide to expanding adequacy and diminishing reactions for certain drugs [4].

\section{HYDROTROPHY}

Hydrotrophy is a solubilization procedure, whereby expansion of a lot of the second solute, the hydrotropic operator brings about an expansion in the fluid solvency of first solute. Hydrotropic operators are ionic natural salts, comprises antacid metal salts of different natural acids [5]. Added substances or salts that expansion dissolvability in given dissolvable is said to "salt in" the solute and those salts that lessening solvency "salt out" the solute. A few salts with huge anions or cations that are themselves solvent in water bring about "salting in" of nonelectrolytes called "hydrotropic salts"; a wonder is known as "hydrotropism." Hydrotrophy assigns the expansion in dissolvability in water because of the nearness of enormous measure of added substances. The instrument by which it improves dissolvability is all the more firmly identified with complexation including a powerless connection between the hydrotropic operators such as sodium benzoate, sodium acetic acid derivation, sodium alginate, urea, and the ineffectively solvent medications.

Hydrotropy is a developing ground-breaking drug solubilization procedure which has been appeared to fundamentally improve the dissolvability of numerous medications. Hydrotropy alludes to the procedure by which a lot of solute improves the dissolvability of another intensify (the medication). They are as often as possible anionic sweetsmelling and non sweet-smelling mixes and can be once in a while impartial [6].

The hydrotropes are known to self-collect in the arrangement. The order of hydrotropes based on atomic structure is troublesome since a wide assortment of mixes has been accounted for to show hydrotropic conduct. Explicit models may incorporate ethanol, sweet-smelling alcohols such as resorcinol, pyrogallol, catechol, $\alpha$ and $\beta$-naphthols and salicylates, and alkaloids such as caffeine and nicotine, and ionic surfactants such as diacids, sodium dodecyl sulfate, and dodecyl oxydibenzene. The fragrant hydrotropes with anionic head gatherings are for the most part contemplated mixes [7]. They are enormous in number in view of isomerism, and their successful hydrotrope activity might be due to the accessibility of intuitive pi $(\pi)$ orbital. 
While recent ultrasound-assisted hydrotropic extraction was used, as a greener approaches for the isolation of geraniol from the leaves of Cymbopogon martini where sodium cumene sulfonate was selected as a suitable hydrotrope for the solubilization studies. It is a clean and green technology, was effectively implemented in extraction of natural products due to advantages like less extraction time, improve in product quality along with reduced processing costs [8].

From the literature survey, it was found that very less work has been done on using natural hydrotropes. The current review focused on advantages and pharmaceutical dosage form using of natural hydrotropes and synthetic hydrotropes. Natural hydrotropes such as caffeine, tannic acid, epigallate, piperazine, and coconut derivatives have been used in few studies. The use of natural hydrotropes is beneficial over synthetic hydrotropes as it is safe for human use. The use of high amount of synthetic hydrotropes is very dangerous for human administration [9].

While using in a combination of natural and synthetic hydrotropes, it has been reported that due to their intermolecular complex formation it helps in enhancing the solubility of drugs.

Hydrotropy is given by the scientist Neuberg Carl A. in 1916. Hydrotropes are basically water-soluble and surface-active compounds; they are useful for enhancing the solubility of organic solutes. They have a molecular phenomenon by adding a second solute (hydrotrope) which is actually beneficial for increasing the aqueous solubility of poorly water-soluble drugs. Hydrotropes always follows an important solubilization technique in which many chemical components said as hydrotropes which used as several-fold to increase in solubility of poorly soluble drugs under normal condition [10].

The hydrotropy acts as increase in the solubility of solutes by the addition of high concentration of alkali metal salts of different organic acids. Which forward by Neuberg, there are several concentrated aqueous hydrotropic agent is available synthetically or naturally. Some of our hydrotropic agents describe as sodium benzoate, niacinamide, citric acid, tannic acid, piperazine, and caffeine have been observed to enhance the aqueous solubility of poorly water-soluble drugs [11].

Hydrotropy is an expansion in water dissolvability brought about by the expansion of a subsequent solute. A few hydrotropic operators, for example, urea, caffeine, sodium benzoate, sodium salicylate, and nicotinamide, have been recognized. The fundamental utilization of this wonder is to build the water solvency of insoluble or somewhat dissolvable medications; there are no reports of the advancement of a hydrotropic plan containing a percutaneous enhancer for the transdermal definition of a water-solvent medication [12]. Unsaturated fat liquor esters are usually utilized as adjuvants for beautifiers and pharmaceuticals. A few esters have been utilized as pervasion enhancers for medications.

The use of a high amount of synthetic hydrotropes is very dangerous for human administration. There has been relatively little work since the introduction of hydrotropy to explore in details and systematic manner the ability of natural hydrotropes to increase the apparent solubility of drug. This research work gives insight to use the natural hydrotropes in solubility enhancement as well as formulation development. Natural hydrotropes can be used in combination of synthetic hydrotropes, and this reduces the concentration of individual hydrotropes using mixed hydrotropy approach [13].

\section{Characteristics of hydrotropes}

- Completely dissolvable in water and essentially insoluble in the framework.

- 2 Hydrotropes are surface dynamic and total in watery arrangement in light of their amphiphilic structure.

- Should not create any temperature when disintegrated in water.

- $\quad$ Cheap and simple accessibility.
- $\quad$ Nonlethal and nonreceptive.

- Insensitive to temperature impacts, when disintegrated in water.

- The dissolvable character being free of $\mathrm{pH}$, high selectivity, and the nonappearance of emulsification are the other one of kind points of interest of hydrotropes.

\section{ADVANTAGES OF HYDROTROPES: [3,13]}

- Hydrotropy is important tool to the other solubilization methods, such as micellar solubilization, salting, and miscibility because of the independent character of solvent.

- Hydrotropes do not require any chemical modification of drugs.

- Hydrotrope should not produce any temperature condition when it dissolved in water.

- It only requires mixing the drug with the hydrotrope in water.

- It does not require chemical modification of hydrophobic drugs, use of organic solvents, or preparation of the emulsion system.

- It provides an efficient, simple, and green platform for various pharmaceutical industries as organic transformations.

- They are easily available and having a low cost.

- Nontoxic and nonreactive.

By the use of hydrotropic agents', the drug solubility may be changed by $\mathrm{pH}$ solution for parenteral administration. The minimum amount of cosolvent is required for the solubility enhancement which should have less toxicity [14]. Parenteral formulation can conduct the problem of reducing injection difficulties and also in blood compatibilities due to increased in viscosity using of cosolvent. These vehicles should contain a less amount and low concentration of cosolvent.

\section{MECHANISM OF HYDROTROPY $[15,16]$}

Each hydrotrope has specific capacity toward a specific segment in the blend, which encourages simple recuperation of the hydrotrope arrangement by controlled weakening with water. Dissolvability does not demonstrate any apparent increment even after the expansion of hydrotrope in the fluid stage, however on ensuing increment in the centralization of hydrotrope, the solvency of the natural compound present in watery stage increments essentially.

This solvency increment in the natural compound when present in water could be because of the development of sorted out gatherings of hydrotrope particles at basic focuses. The basic convergence of hydrotrope is named as the base hydrotrope fixation minimum hydrotropic concentration (MHC) which is the base required hydrotrope focus in the watery stage above which the dissolvability of the natural compound in fluid stage increments altogether.

This expansion is apparently through a self-collection process due to their amphiphilic nature and differs with the idea of the natural compound. The expanding pattern is kept up just up to a specific grouping of hydrotrope past which there is no calculable increment in the dissolvability of natural compound in the watery stage. This convergence of hydrotrope is named as the greatest hydrotrope focus.

Barely any models important to the system of hydrotropy are exhibited beneath: The plant cell divider is comprised of phospholipid bilayers [17]. The hydrotrope demolishes the phospholipid bilayer and infiltrates through the cell divider into the inward structures. The water drenching indicates exceptionally less impact on stopper cells. The cellulose and suberin lamellae are the cell divider part of plug cells. The suberin lamella makes the plug cell impermeable to water. In any case, the hydrotrope arrangements tear open the water-impermeable suberin lamella and after that the develop stopper cells. The plug cell layers are bothered by the hydrotrope and the watery arrangement enters through the cell divider. At the point when the inward part is presented to the hydrotrope arrangement, the cell swells and liberates the cells from firmly bound structures. Hydrotropic arrangements accelerated the solutes; out of the arrangement on weakening with water in this manner empower the prepared recuperation of the 
disintegrated solutes. Hydrotropic operators can make the $\mathrm{O} / \mathrm{W}$ and $\mathrm{W} / 0$ microemulsion and the lamellar fluid precious stone destabilized, which results in the "stage change" from lamellar fluid gem stage to biconstant structure this is called as hydrotrope-solubilization activity. Nutrient C demonstrates hydrotrope-solubilization activity [19].

Hydrotropes are known as "coupling specialists.". At the point when hydrotropes are added to a turbid fluid with generally high water substance makes the fluid straightforward in light of stage change.

\section{Self-association of hydrotropes}

As of late, self-collection of hydrotropes in fluid medium has been accounted for by various laborers. The term least hydrotrope focus (MHC) has been utilized in consonance with the critical micelle concentration (CMC) of surfactants. Rath has accentuated the development of intermolecular stack-type collection of hydrotropes. Like Rath, they have additionally considered stacking of hydrotropes as the procedure for self-affiliation. From point by point physicochemical investigations, Balasubramanian and collaborators, presumed that hydrotrope activity is a group sub-atomic wonder including the arrangement of non-covalent totals. As indicated by them, there are comparable microenvironmental features of hydrotrope assemblies with micelles.

- Surface activity of hydrotrope solutions is similar to micelle forming surfactants

- Sigmoidal solubilization curves show cooperativity in the process, and

- Open-layer assemblies of hydrotropes exist in their crystalline state.

The high focus required for self-relationship of hydrotropes (for example, high MHC values) emphatically differentiates micelle arrangement and throws questions on the development of requested congregations as in micelles [20]. Furthermore, the hydrotropes are basically not effective surface-dynamic mixes, for example, they do not considerably bring down the surface pressure of water. The hydrotropes have delivered tedious change in the warm conduct; no sharp change has been watched. The self-relationship of hydrotropes yielding a break at higher solute fixation is along these lines suspicious; this can be a typical event of a deliberate property because of action and different impacts. Further work is required for a definitive choice on hydrotrope affiliation. Up to that point, the idea of hydrotrope affiliation and MHC must not be disposed of, yet ought to be utilized with reservation $[21,22]$.

\section{Readiness of hydrotropes}

Hydrotropes are created by sulfonation of a fragrant hydrocarbon dissolvable (i.e., toluene, xylene, or cumene) [23]. The subsequent fragrant sulfonic corrosive is killed utilizing a fitting base (e.g., sodium hydroxide) to create the sulfonate or hydrotrope. The hydrotropes are "unadulterated" substances yet are delivered and moved in either fluid arrangements, normally at a 30-60\% degree of action, or in granular solids commonly at $90-95 \%$ degree of action. Different segments of granular solids incorporate sodium sulfate and water. Fluid item is created in a shut framework. Granular hydrotropes item is created by shower drying that incorporates source control and residue accumulation $[25,26]$. Hydrotropes are made for mechanical/expert and shopper use and are not utilized as intermediates/subordinates for further compound assembling procedures or employments.

A hydrotrope is an intensify that solubilizes hydrophobic mixes in watery arrangements. Ordinarily, hydrotropes comprise a hydrophilic part and a hydrophobic part (like surfactants), yet the hydrophobic part is commonly too little to even consider causing unconstrained self-conglomeration [27]. Hydrotropes do not have a basic focus above which self-accumulation "all of a sudden" begin to happen. Rather, some hydrotropes total in a stage shrewd self-conglomeration process, bit by bit expanding accumulation size. In any case, numerous hydrotropes do not appear to self-total by any means, except if a solubilizate has been included [28]. To improved the fluid dissolvability of medication, an inadequately water-solvent medication by utilization of concentrated arrangement of urea (a hydrotropic operator).

Hydrotropy is suggested to be superior to the other solubilization methods, such as miscibility, micelles solubilization, cosolvency and salting in, because of the independent character of ph has highly selected. It only requires the mixing of drug with the hydrotropes in water $[29,30]$.

The hydrotropic agents who are present in the synthetic and natural form which are defined as non-micelle- forming substances either liquids or solids, organic and in organic, which is capable of solubilizing insoluble compounds. According to various review of literature shows that a large number of poorly water-soluble drugs have been analyzed, namely, furosemide, cefixime, salicylic acid, piroxicam, ketoprofen, aceclofenac, aspirin, hydrochlorothiazide, naproxen, and paracetamol using hydrotropic solubilizing agent $[30,31,38]$.

There are various large number of hydrotropic agents have been employed to enhance aqueous solubility of various poorly watersoluble drugs [39]. While mixed solvency concept were used in various formulation development of syrups such as in liquid oral solution development of syrup of poorly water soluble. This approach shall prove a boon in pharmaceutical field to develop various formulations of poorly water-soluble drugs combining various water-soluble excipients in safe concentration to produce a desirable aqueous solubility of poorly water-soluble drug [40].

While using a synthetic hydrotropic agent such as sodium benzoate, urea, nicotinamide, citric acid, and salicylic acid, while using them in high concentration it may cause toxicity, which is harmful to human beings [40].

Demonstration of natural hydrotropes while enhancing the solubility of poorly soluble drugs which are naturally occurring as caffeine, piperazine, tannic acid, and epigallocatechin gallate so that it involves the use of low concentration of them. Moreover, it helps in overcome environmental and energy issues along with the mentioned one for conventional method.

The natural hydrotropes processing can be considered as a green extraction since they are artificially dormant, effectively distinguishable, promptly reusable, and particular toward focused mixes. Be that as it may, this technique experiences the constraint of expanded extraction time and a higher grouping of hydrotropes $[10,40]$.

Dissolvability investigations of drug in various solvents: Solubility study was performed by the shake flask method. The abundance tranquilize alongside various solvents was taken in $10 \mathrm{ml}$ stoppered volumetric cups. The flagons were liable to shaking in an orbital shaker for $24 \mathrm{~h}$ at $25^{\circ} \mathrm{C}$ and $60 \mathrm{rpm}$ speed and further equilibrate for next $24 \mathrm{~h}$. At that point, the arrangements were centrifuged at $1000 \mathrm{rpm}$ for $10 \mathrm{~min}$ and separated through Whatman channel paper $(0.45 \mu \mathrm{m})$. The absorbance record on ultraviolet (UV) spectrophotometer of these channels arrangements after fitting weakenings to decide the dissolvability utilizing separate solvents as clear $[4,16,40]$.

The $\mathrm{pH}$ subordinate dissolvability: All the examples of immersed arrangement of medication at various $\mathrm{pH}$ were shaken in an orbital shaker for $24 \mathrm{~h}$ at $25^{\circ} \mathrm{C}$ and $60 \mathrm{rpm}$ and further equilibrated for next 24 h. After centrifugation and filtration, the absorbance was recorded at UV range utilizing individual arrangements as clear [41].

Dissolvability with various hydrotropes: Solubility of etodolac in different arrangements was dictated by shake flask method. Overabundance measure of medication was added to $10 \mathrm{ml}$ stoppered volumetric carafes. Different hydrotropes in $20 \%$ and $40 \%$ focus arrangements in refined water were filled in the volumetric jars. The 
flagons were shaken for $12 \mathrm{~h}$ in an orbital shaker at $25^{\circ} \mathrm{C}$ and $60 \mathrm{rpm}$ speed. The arrangements were permitted to equilibrate for the following $24 \mathrm{~h}$. The arrangements were then centrifuged for $10 \mathrm{~min}$ at $1000 \mathrm{rpm}$. Supernatants of each example were sifted through $0.45 \mu \mathrm{m}$ film channel and dissected for medication content spectrophotometrically at UV goes after appropriate weakenings [41].

\section{Different strategies to gauge the solubility}

To decide the dissolvability of solids in fluids following two stages are utilized [41].

1. Preparation of soaked arrangement: Solubility demonstrates the most extreme measure of a substance that can be broken up in a dissolvable at a given temperature. Such an answer is called soaked. Dissolvability is estimated either in grams per $100 \mathrm{~g}$ of dissolvable (g/100 g) or number of moles per $1 \mathrm{~L}$ of the arrangement.

2. Analysis of immersed arrangement: Once the soaked arrangement is readied its examination is completed to check the solvency. It relies on the idea of the solute and precision of the strategy utilized. Following strategies are utilized for investigation.

A. Evaporation technique

B. Volumetric technique

C. Gravimetric technique

D. Instrumental technique.

Assurance of importance of hydrotropic polymer in the spectrophotometric estimation of drugs.

An UV-visible chronicle spectrophotometer with $1 \mathrm{~cm}$ coordinated silica cells was utilized for spectrophotometric judgments. For assurance of impedance of hydrotropic specialists in the spectrophotometric estimation of the standard arrangements of medications was resolved in refined water alone and within the sight of the most extreme centralization of the hydrotropic operator utilized for spectrophotometric investigation.

The absorbances were recorded against particular reagent spaces at fitting wavelengths. Titrimetric investigation strategy utilized for deciding balance dissolvability at room temperature. Upgrade proportions in solubilities were dictated by the following equation -

Upgrade proportion=Solubility in hydrotropic arrangement/Solubility in refined water

\section{Hydrotropy and hydrotropic operators $[6,12,34]$}

In 1916, hydrotropy was designated by the researcher Cral A. Neuberg. Hydrotropes go about as an amphiphilic sub-atomic structure has the capacity to build the solvency of water inadequately dissolvable medication. Basically the nearness of enormous amount of one solute improves the solvency of another solute. Fundamentally hydrotropic operators are expressed as ionic natural salt which increments or diminish the solute in given dissolvable as salt in or salt out impact. Hydrotropes contain both hydrophobic and hydrophilic fractions in them. The viability of hydrotrope solubilization relies on the balance among hydrophobic and hydrophilic part of hydrotropes.

The bigger measure of the hydrophobic part is utilized as added substances, gives better hydrotropic productivity. The hydrotropic agents can be aromatic anionics which include sodium benzoate, sodium salicylate, sodium benzene sulfonate, sodium benzene disulfonate, sodium para toluene sulfonate, and sodium cumene sulfonate, another aromatic cationic examples - para aminobenzoic acid hydrochloride, procaine hydrochloride, and caffeine, and neutral, organic and inorganic liquids or solid in nature.

Applications of hydrotropic solubilization in various fields of pharmacy $[44,45]$

Hydrotropes are important tool in the both academic and pharmaceutical industry. They vary many realistic applications in both biomedical sciences and engineering fields also. There uses are play an important parameters in the pharmaceutical formulations solute separations process, selective separation and alternation in reaction kinetics. Various applications are discussed below which related to development of pharmaceuticals.

- Quantitative determination of poorly water-soluble drugs by titrimetric analysis such as naproxen, aspirin, furosemide, ibuprofen, salicylic acid, famotidine, and aceclofenac using different hydrotropic agents such as sodium benzoate, and nicotinamide.

- Manufacturing of solid dispersions of poorly soluble drugs pre including the use of organic solvents. Carvedilol using b-cyclodextrin; celecoxib using b-cyclodextrin; carbamazepine using hydrotropic solubilization nicotinamide; and halofantrine using polymers PVP800.

- Application of hydro solubilization in the extraction of pure constituents from crude drugs.

- Applications of mixed solvency to broadly develop injection dosage forms of poorly water-soluble drugs.

- The use of hydrotropy gives an instant release of poorly soluble drugs from the suppositories.

- The use of hydrotropic solubilizers as permeation enhancers.

- Used in manufacturing of syrups (for reconstitution) of poorly watersoluble drugs.

- Quantitative analysis and by titrimetric analysis preincluding the use of organic solvents.

\section{Parenteral formulations [46]}

The parenteral formulation has numerous benefits in drug discovery and development. It enables efficacy, toxicity, and pharmacokinetics studies. Parenteral products should provide various advantages such as rapid onset of action with good bioavailability, improve soundness of medications, and lessen dosing recurrence. Progressively reliable dosing can be accomplished by decreasing nourishment impact through details. An intramuscular medicine is given by needle into the muscle. It tends to be the arrangement, suspension or oil. In parenteral, the vehicle ought to contain a base sum and low centralization of the codissolvable to lessen thickness and harmfulness impact. Novel parenteral conveyance frameworks, for example, nanoparticles, patches are likewise accessible now for controlled and dynamic focused on medication conveyance.

Antibiotics aqueous injection was prepared using mixed solvency approaches (sodium benzoate, urea, sodium citrate, sodium acetate, and nicotinamide). The formulation of the aqueous injection gives antimicrobial effectiveness and stability. The mixed solvency approaches are useful in solubility enhancement of drug without affecting its antimicrobial properties.

Other parenteral administration prepares using hydrotropic solubilization to elucidate the probable mechanism of solubilization. Parenteral formulations using piperazine as hydrotrope developed and show better physical and chemical stability [46].

The effect of caffeine and nicotinamide as a hydrotropic agent was used for the solubility of the Vitamin $B_{2}$ at aqueous solution.

\section{Transdermal formulations}

Transdermal formulation offers to the likelihood of delivering supported plasma medication levels and of evading hepatic firstpass digestion. Numerous mixes, for example, unsaturated fats, propylene glycol, amines, and amides are known to upgrade tranquilize penetration. Pharmaceutical transporter, for example, blended dissolvability, codissolvable frameworks is ordinarily joined enhancers into transdermal plan. Transdermal definition was readied utilizing unsaturated fat monoesters as hydrotropic operator. Hydrotropic transdermal definition improved skin saturation due its capacity of hydrotrope to shape totals. Paraben containing semisolid topical detailing gets ready with nicotinamide which lessens stratum corneum vehicles parcel coefficient. They lessen the toxicological hazard too.

Recently revealed solubilization of polyol unsaturated fat monoesters in water within the sight of diclofenac sodium, bringing about 
enhance solubility of diclofenac. In this examination, we analyzed two hydrotropic salts, sodium salicylate, and sodium benzoate, for their capacity to solubilize polyol unsaturated fat esters in water. Close infrared spectrophotometry and 1H NMR spectroscopy were utilized to explore the physicochemical properties of the hydrotropic framework. What is more, we thought about the improvement impacts of polyol unsaturated fat esters in a hydrotropic framework with those acquired in codissolvable frameworks and blended micelles.

Gels plan by starch without warmth salicylate as a gelling operator, and discharge examples of the gels were examined utilizing drug as a model medication. Medication may be indicated as reliable dispersion controlled energy. Examples of the medication discharge relied on the underlying stacking levels and the starch substance of the gels. Hence, hydrotrope-gelled starch demonstrated to be a superior vehicle for topical medication delivery $[6,23,46]$.

\section{Natural hydrotropes: [46]}

- The effectively solubilization of hydrophobic drugs using catechin gallate or tannic acid is useful for increased in the aqueous solubility of all drugs. The use of these additives may provide improved formulation of various hydrophobic drugs using oral, parenteral, localized or device associated delivery system.

- The potential mechanism for hydrotropic solubilization of riboflavin and caffeine experience atomic parallel stacking in the fluid arrangement, the outcome connecting near oneself stacking of caffeine under similar conditions.

- Effect of a blend of caffeine and nicotinamide on the regular blend of the hydrotropic mix on the solvency of nutrient in watery arrangement. It is discovered that a blend of two hydrotropic specialists prompts an expansion in the solvency of the nutrient, instead of utilizing only one hydrotropic operator it is recommended that a procedure for improving the dissolvability of fragrant medications is to utilize a blend of hydrotropic operators.

- Caffeine and nicotinamide improve the watery dissolvability of the counter malarial operator halofantrine. Solubility is expanded to a more prominent degree within the sight of caffeine $(12.5-125 \mathrm{~nm})$ than nicotinamide $(125 \mathrm{~nm}-2.0 \mathrm{M})$.

\section{The potential for hydrotropy [47]}

The progress in hydrotropy has boosted their use in various operational fields there is growth on advance using application of machine learning prediction enhancing solubility potential along with a non-toxic effect, ecofriendly, and nonflammable. The progress in hydrotropy as well as novel drug delivery system and mechanism of hydrotropic drugs with their compatibilities and biocompatibilities. This technology is further expected to transform the advances toward the using machine learning in studies of hydrotrope enhanced solubilization of poorly aqueous soluble drugs as well as critical moieties with narrow therapeutic index. Through the use of natural hydrotropes, we formulated various pharmaceutical dosage forms.

\section{Application of hydrotropic agent in the pharmaceutical field and industry: [47]}

- Preparation of dry syrups (for reconstitution) of ineffectively watersolvent medications.

- Quantitative estimations of ineffectively water dissolvable medications by UV-Visible spectrophotometric investigation blocking the utilization of natural solvents.

- Quantitative estimations of inadequately water solvent medications by the titrimetric investigation, for example, ibuprofen, flurbiprofen, and naproxen utilizing sodium benzoate.

- Preparation of hydrotropic strong scatterings of inadequately waterdissolvable medications blocking the utilization of natural solvents, for example, felodipine utilizing poly-ethylene glycol 6000 and polyvinyl liquor.

- Hydrotropic arrangements can likewise be attempted to build up the disintegration liquids to complete the disintegration investigations of dose types of ineffectively water-solvent medications.

- The utilization of hydrotropic solubilizers as pervasion enhancers.
- Application of hydrotropic solubilization in nanotechnology (by controlled precipitation).

- Application of hydrotropic solubilization in the extraction of dynamic constituents from unrefined medications (in pharmacognosy field).

- Hydrotropes have been utilized to solubilize natural mixes, colors, drugs, and bio-synthetic compounds.

- Hydrotropes have been tried in the advancement of extractive partition forms in the detachment of proteins and refining as an extractive dissolvable for the division of close breaking point phenolic blends.

- Aqueous hydrotrope arrangements give protected and compelling media to the extraction of common items and for leading natural manufactured responses.

- Hydrotropes find wide applications in, cleanser definition, social insurance, and family unit purposes.

- They have been utilized to build the pace of heterogeneous responses.

- They are utilized as an extraction operator for aromas.

- Hydrotropic solubilization in nanotechnology (by controlled precipitation).

- Hydrotropy to give quick arrival of ineffectively water-dissolvable medications from the suppositories.

- Used in the planning of boring admirably liquids and the division of water-oil emulsion.

- It may be utilized in the oil business, in tertiary oil recuperation just as in different procedures.

- Hydrotropes change the thickness of surfactant plans and increment the cloud purpose of cleansers.

- Aqueous hydrotrope arrangements give sheltered and viable media to the extraction of characteristic items and for leading natural engineered responses.

- Viscosity and cloud point (the temperature at which a reasonable item starts to end up foggy after cooling) of fluid cleansers can be constrained using hydrotrope specialists.

- Hydrotropes improve the strength of the concentrated fluid cleansers by upgrading the solvency of the surfactants and by managing the gelling propensity which fluid cleansers can display on weakening with water

- This procedure might be utilized to recoup the solute in the crystalline structure at an improved immaculateness, and the rest of the mother alcohol could be utilized to focus the hydrotrope for reusing.

- Hydrotropes have been connected in, shampoos, degreasing mixes, and printing glues, as an added substance for pastes utilized in the calfskin business.

- The utilization of hydrotrope, sodium xylene sulfonate in paper mash producing industry gives magnificent outcomes.

\section{Blended hydrotropy [47]}

Blended hydrotropic solubilization method is the marvel to build the solvency of ineffectively water-dissolvable medications in the mixes of hydrotropic operators, which may give phenomenal synergistic upgrade impact on dissolvability of inadequately water solvent medications, usage of it in the detailing of measurements types of water-insoluble medications and to decrease grouping of individual hydrotropic specialist to limit the reactions instead of utilizing an enormous centralization of one hydrotrope a mix of, state, five hydrotropes can be utilized in 1 /fifth focuses diminishing their individual toxicities.

Favorable circumstances of blended hydrotropic solubilization [48]

1. It may decrease the huge all out grouping of hydrotropic operators important to deliver unobtrusive increment in solvency by utilizing a mix of specialists in lower focus.

2. It is new, straightforward, practical, protected, exact, exact and ecological well-disposed strategy for the examination (titrimetric and spectrophotometric) of ineffectively water-dissolvable medications titrimetric and spectrophotometric blocking the utilization of natural solvents.

3. It blocks the utilization of natural solvents and along these lines dodges the issue of lingering poisonous quality, mistake because of unpredictability, contamination, cost, and so forth. 


\section{OTHER TECHNIQUES FOR SOLUBILITY ENHANCEMENT $[17,22,36]$}

Solubility improvement strategies can be classified into physical change, concoction alterations of the medication substance, and different methods.

- Physical modifications: Particle size decrease such as micronization and nanosuspension, adjustment of the gem propensity such as polymorphs, undefined structure, and cocrystallization, tranquilize scattering in bearers such as eutectic blends, strong scatterings, strong arrangements, and cryogenic methods.

- Compound modifications: Change of ph, utilization of buffer, derivatization, complexation, and salt development.

- Different methods: Supercritical liquid procedure, utilization of adjuvants such as surfactant, solubilizers, cosolvency, and novel excipients.

Table 1: The United States Pharmacopeia describes solubility using the seven groups listed $[11,18,40]$

\begin{tabular}{ll}
\hline Descriptive term & Part of solvent required per part of solute \\
\hline Very soluble & $<1$ \\
Freely soluble & From 1 to 10 \\
Soluble & From 10 to 30 \\
Sparingly soluble & From 30 to 100 \\
Slightly soluble & From 100 to 1000 \\
Very slightly soluble & From 1000 to 10,000 \\
Practically insoluble & 10,000 and over \\
\hline
\end{tabular}

Particle size reduction

The solvency of medication is frequently inherently identified with medication molecule size; as a molecule decreases, the surface region to volume proportion increments. The bigger surface region permits more prominent association with the dissolvable which causes expansion in solvency [48].

\section{Hydrotropes as medication bearer}

Hydrotropes can possibly go about as carriers for dynamic pharmaceutical fixings. They have the capacity to create dynamic, noncovalent congregations, for example, groups in fluid arrangements. Within the sight of hydrophobic aggravates, these groups are balanced out by arrangement of seemingly perpetual, profoundly stable mesoscopic beads because of a wonder known as "mesoscale solubilization." Such materials can help in procedure in different items going from pharmaceuticals, beautifiers, and agrochemicals [49]. Unpretentious changes in surfactant geometry lead to a stamped impact on the plainly visible rheological conduct of the framework. These micellar arrangements go about as a layout for tissue building and as a modifier of the medication conveyance [50]. Furthermore, hydrotropes assume different jobs such as o/w microemulsion stabilizers, thickness modifiers, cleaning specialists, and solubilizers in detailing advancement.

\section{Solid dispersions}

Solid dispersions speak to a helpful pharmaceutical procedure for expanding the disintegration, retention, and restorative viability

Table 2: Biopharmaceutical classification system [22,38]

\begin{tabular}{llll}
\hline Class & Solubility & Permeability & Drugs \\
\hline 1 & Highly soluble & Highly permeable & Diltiazem, verapamil, metoprolol \\
2 & Poorly soluble & Highly permeable & Piroxicam, carbamazepine, glibenclamide \\
3 & Highly soluble & Poorly soluble & Metformin, captopril, cimetidine \\
4 & Poorly soluble & Poorly permeable & Furosemide, hydrochlorothiazide, Ritonavir \\
\hline
\end{tabular}

Table 3: Some important implementation of hydrotropes in the formulation and their key roles example of oral formulation for which hydrotropic agents are used $[45,46]$

\begin{tabular}{lll}
\hline Drug & Hydrotropic agent & Key finding \\
\hline Aceclofenac & Urea 20\% and sodium citrate 10\% & 1.7 fold improvement in vivo dissolution \\
Mefenamic acid & 0.5 M sodium citrate & Improvement in dissolution studies has shown a 62\% increase in solubility \\
Furosemide & $15: 20: 5$ urea, sodium benzoate, sodium citrate & Improvement in the solubility of furosemide. \\
Nevirapine & Citric acid 10\% and urea 20\% & Enhancement in solubility of nevirapine \\
Telmisartan & Urea 10\% & 15737 folds improved solubility \\
\hline
\end{tabular}

Table 4: Some examples of parenteral formulation using various hydrotropic agents $[32,46]$

\begin{tabular}{lll}
\hline Drug & Hydrotropic agent & Key finding \\
\hline Zaltoprofen & Urea, sodium benzoate & Improved in solubility more than 600 fold \\
Aceclofenac & Urea, sodium citrate & Improved in solubility more than 250 folds \\
Aceclofenac & Sodium citrate, nicotinamide, urea & Improved in solubility more than 200 folds \\
Riboflavin & Caffeine, nicotinamide & Two hydrotropic agents lead to increase in the solubility of vitamin in three components \\
Etodolac & Sodium benzoate, sodium acetate & 15\% sodium benzoate and 25\% sodium acetate were found to be more stable \\
Nimesulide & Piperazine, sodium benzoate & Improved in solubility using piperazine as hydrotrope was developed \\
Indomethacin & Arginine & Aqueous formulation using arginine as hydrotrope was developed \\
Ofloxacin & Sodium citrate, sodium benzoate, urea & Aqueous injection of drug was prepared for antimicrobial effectiveness and stability \\
Halofantrine & Caffeine, nicotinamide & Solubility is increased to a greater extent in the presence of caffeine (12.5-125mM) \\
\hline
\end{tabular}

Table 5: Example of spectrophotometric and oral formulation for which hydrotropic agents are used [31, 36, 47]

\begin{tabular}{llll}
\hline Drug & Formulation & Hydrotropic agent & Increase in solubility \\
\hline Hydrochloric thiazide & Tablets & $40 \%$ niacinamide, sodium acetate & 25 -folds \\
Paracetamol & Tablets & $5 \mathrm{M}$ urea & 22 -folds \\
Terconazole & Bulk form & urea & Values increase in the amount of urea \\
Indomethacin & Capsules & $2 \mathrm{M}$ niacinamide & $99.33-101.04$. \\
Aceclofenac & Bulk form and tablets & $30 \%$ urea 20\% sodium citrate & 0.2735 \\
\hline
\end{tabular}


of medications in measurement structures [51]. The term strong scattering alludes to a gathering of strong items comprising in any event two distinct segments, by and large a hydrophilic grid and a hydrophobic medication. The most generally utilized hydrophilic bearers for strong scatterings incorporate polyvinylpyrrolidone (Povidone, PVP), polyethylene glycols, and Plasdone-S630. Surfactants such as Tween-80, docusate sodium, Myrj-52, Pluronic-F68, and sodium lauryl sulfate likewise discover a spot in the detailing of strong scattering [52].

\section{Nanosuspension}

Nanosuspension innovation has been created as a promising contender for efficient conveyance of hydrophobic medications. This innovation is connected to inadequately solvent medications that are insoluble in both water and oils. A pharmaceutical nanosus-benefit is a biphasic framework comprising nano estimated tranquilize particles balanced out by surfactants for either oral and topical use or parenteral and aspiratory organization. The particle size conveyance of the strong particles in nanosuspensions is generally short of what one micron with a normal molecule size going somewhere in the range of 200-600 nm.

\section{Supercritical fluid (SCF) process}

Another tale nanosizing and solubilization innovation whose application has expanded as of late is molecule size decrease by means of supercritical liquid (SCF) forms. Super-basic liquids are liquids whose temperature and weight are more prominent than its basic temperature (Tc) and basic weight (Tp), enabling it to accept the properties of both a fluid and a gas. At close basic temperatures, SCFs, are exceedingly compressible enabling moderate changes in strain to significantly adjust the thickness and mass vehicle qualities of the liquid that to a great extent decide its dissolvable power. When the medication particles are solubilized inside the SCF (more often than not carbon dioxide), they might be recrystalized at enormously decreased molecule sizes [53]. The adaptability and accuracy offered by SCF procedures permit micronization of medication particles inside tight scopes of molecule size, regularly to submicron levels. Current SCF procedures have exhibited the capacity to make nanoparticulate suspensions of particles 5-2000 $\mathrm{nm}$ in width.

\section{Cryogenic techniques}

Cryogenic procedures have been created to upgrade the disintegration pace of medications by making nanostructured love photos tranquilize particles with high level of porosity at extremely low-temperature conditions. Cryogenic creations can be characterized by the sort of infusion gadget (slender, rotational, pneumatic, and ultrasonic spout), area of spout (above or under the fluid level), and the organization of cryogenic fluid (hydrofluoroalkanes, N2, $\mathrm{Ar}, \mathrm{O}_{2}$, and natural solvents) [54]. After cryogenic handling, dry powder can be acquired by different drying procedures such as splash stop drying, barometrical stop drying, vacuum stop drying, and lyophilization.

\section{Incorporation complex formation-based techniques}

Incorporation complex development system has been utilized all the more definitely to improve the fluid solvency, disintegration rate, and bioavailability of inadequately water solvent medications. Incorporation buildings are framed by the inclusion of the nonpolar particle or the nonpolar district of one atom (known as a visitor) into the hole of another particle or gathering of particles (known as host). The most regularly utilized host atoms are cyclodextrins [55].

\section{Micellar solubilization}

The utilization of surfactants to improve the disintegration performance of inadequately solvent medication items is likely the fundamental, essential, and the most seasoned technique. Surfactants decrease surface pressure and improve the disintegration of lipophilic medications in a watery medium. They are additionally used to balance out medication sus-annuities. At the point when the centralization of surfactants surpasses their basic micelle focus (CMC, which is in the scope of $0.05-0.10 \%$ for most surfactants), micelle arrangement happens which ensnare the medications inside the micelles. This is known as micellization and for the most part brings about upgraded dissolvability of inadequately solvent medications. Surfactant additionally improves wetting of solids and builds the pace of deterioration of strong into better particles $[55,56]$.

\section{Crystal engineering}

The surface territory of medication accessible for disintegration is define-gouge on its molecule size and capacity to be wetted by luminal liquids. This molecule size, which is basic to sedate disintegration rate, is reliant on the states of crystallization or on techniques for comminution, for example, sway processing and liquid vitality processing [55].

\section{CONCLUSION}

By this study, we can reason that solubility is the most significant physical normal for a medication for its oral bioavailability, definition, advancement of various measurements type of various medications, and for quantitative examination. Solvency can be upgraded by numerous procedures among them hydrotropy is of particular significance. Hydrotropy is implying a solubilization procedure whereby expansion of a lot of second solutes brings about an increment in the watery solvency of another solute and the synthetic compounds which are utilized in hydrotropy are called hydrotropes. For instance sodium benzoate, urea, sodium salicylate, and ibuprofen sodium and so forth, solubility can be improved by numerous systems and number of folds increment in dissolvability is accounted for as well. In view of dissolvability issue of numerous medications the bioavailability of them gets influenced, and henceforth solvency improvement winds up fundamental. It is presently conceivable that to build the solvency of inadequately solvent medications with the assistance of different systems as referenced previously. In present situation, this technique is getting part of qualities and might be demonstrated the best strategy in future.

\section{AUTHORS' CONTRIBUTIONS}

The authors are thankful to Sardar Bhagwan Singh University for providing their support and necessary facilities to carry out the review work.

\section{CONFLICTS OF INTEREST}

Nil.

\section{REFERENCES}

1. Agrawal S, Pancholi SS, Jain NK, Agrawal GP. Hydrotropic solubilization of nimesulide for parenteral administration. Int J Pharm 2004:274:149-55.

2. Badwan AA, El-Khordagui LK, Saleh AM, Khalil SA. The solubility of benzodiazepines in sodium salicylate solution and a proposed mechanism for hydrotropic solubilization. Int J Pharm 1982;13:34-41.

3. Beig A. Hydrotropic solubilization of lipophilic drugs for oral delivery: The effect of urea and nicotinamide on carbamazepin solubility- permibility. Front Pharmacol 2011;1:19-25.

4. Cui Y. Hydrotropic solubilization by urea derivatives: A molecular dynamics simulation study. J Pharm (Cairo) 2013;2013:791370.

5. Shukla R, Patel A, Soni M, Modi V. Quantitative spectrophotometric estimation of cefadroxil using hydrotropic solubilization technique. Asian J Pharm 2008;2:146-7.

6. Maheshwari RK, Srivastav VK, Prajapat RP, Jain A, Kamaria P, Sahu S. New spectrophotometric estimation of ornidazole tablets employing urea as a hydrotropic solubilizing additive. Indian J Pharm Sci 2010;72:258-61.

7. Maheshwari R, Rajput M, Sinha S. Ecofriendly spectrophotometric estimation of tinidazole in tablets using lignocaine HCL as a hydrotropic solubilising agent. Asian J Pharm 2009;3:319-21.

8. Dandekar D, Gaikar V. Hydrotropic extraction of curcuminoids from turmeric. Sep Sci Technol 2003;38:1185-215.

9. Padalkar K, Gaikar V. Extraction of piperine from piper nigrum (black pepper) by aqueous solutions of surfactant and surfactant + hydrotrope mixtures. Sep Sci Technol 2008;43:3097-118 
10. Ansari K, Gaikar V. Green hydrotropic extraction technology for delignification of sugarcane bagasse by using alky benzene sulfonates as hydrotropes. Chem Eng Sci 2014;115:1157-66.

11. Gaikar VG, Sharma MM. Note: Extractive separations with hydrotropes. Solvent Extr Ion Exc 1986;4:839-46.

12. Bhat M, Gaikar V. Characterization of interaction between butyl benzene sulfonates and cetyl trimethyl ammonium bromide in a mixed aggregate systems. Langmuir 1999;5:4740-51.

13. Rathod M, Agarwal S. Development and evaluation of furosemide microspheres made by mixed solvency concept. Int J Pharm Erudition 2013;2:22-31

14. Evstigneev MP, Evstigneev VP, Santiago AA, Davies DB. Effect of a mixture of caffeine and nicotinamide on the solubility of Vitamin (B2) in aqueous solution. Eur J Pharm Sci 2006;28:59-66.

15. Gadade DD, Lohade TS, Lahoti SR, Rawat SS, Maheshwari RK. Solubility enhancement of ofloxacin by mixed solvency approaches. Indian Drugs 2018;55:34-40.

16. Girishpai K, Divya S, Reddy MS, Kumar L, Krishna TV. Solubility enhancement of norfloxacin by hydrotropy technique. Int J Pharm Pharm Sci 2014;6:395-7.

17. Jain N, Jain R, Jain DK, Maheshwari RK, Jain S. Novel UVspectrophotometric method for quantitative estimation of furazolidone using mixed hydrotropic agent. Pak J Pharm Sci 2013;26:159-62.

18. Jackson JK, Letchford K. The effective solubilization of hydrophobic drugs using epigallocatechin gallate or tannic acid-based formulations. J Pharm Sci 2016;105:3143-52

19. Kumar SV, Raja C, Jayakumar C. A review on solubility enhancement using hydrotropic phenomena. Int J Pharm Sci 2014;6:1-7.

20. Kate BA, Phulzalke SB, Deshmukh MT. Solubility enhancement of poorly water soluble drug zaltoprofen by mixed hydrotropy approaches. Asian J Biomed Pharm Sci 2016;6:32-9.

21. Damiati SA, Martini LG, Smith NW, Lawrence MJ, Barlow DJ. Application of machine learning in prediction of hydrotrope-enhanced solubilisation of indomethacin. Int J Pharm 2017;530:99-106.

22. Dhapte V, Mehta PP. Advances in hydrotropic solution: An update review. St. Petersburg Polytechn Univ J 2016;1:424-35.

23. Lim LY, Go ML. Caffeine and nicotinamide enhances the aqueous solubility of the antimalarial agent halofantrine. Eur J Pharm Sci 2000;10:17-28.

24. Limbird LE, Hardman JG. The Pharmacological Basis of Therapeutics. $10^{\text {th }}$ ed. New York: McGraw-Hill Medical Publishing Division; 2001.

25. Kumar Maheshwari R, Indurkhya A. Formulation and evaluation of aceclofenac injection made by mixed hydrotropic solubilization technique. Iran J Pharm Res 2010;9:233-42.

26. Madan JR, Pawar KT, Dua K. Solubility enhancement studies on lurasidone hydrochloride using mixed hydrotropy. Int J Pharm Investig 2015;5:114-20.

27. Madan JR, Kamate VJ, Dua K, Awasthi R. Improving the solubility of nevirapine using A hydrotropy and mixed hydrotropy based solid dispersion approach. Polim Med 2017;47:83-90.

28. Maheshwari RK, Jagwani Y. Mixed hydrotropy: Novel science of solubility enhancement. Indian J Pharm Sci 2011;73:179-83.

29. Maheshwari RK, Rathore A, Agrawal A, Gupta MA. New spectrophotometric estimation of indomethacin capsules with niacinamide as hydrotropic solubilizing agent. Pharm Methods 2011;2:184-8

30. Maheshwari Y, Mishra DK, Mahajan SC, Maheshwari P, Maheshwari RK, Jain V. Novel pharmaceutical application of mixed solvency in the formulation development of syrups (Liquids Oral Solution) of poorly water- soluble drugs. Int J Pharm Sci 2013;3:753-8.

31. Maheshwari RK, Rajput MS, Sharma S, Nair V. New spectrophotometric of hydrochlorothiazide in tablets using mixed hydrotropic solubilization technique. Sch Res Lib 2010;2:70-4.

32. Nidhi K, Indrajeet S, Khushboo M, Gauri K, Sen DJ. Hydrotropy: A promising tools for solubility enhancement: A review. Int J Drug Dev Res 2011;5:32-9.

33. Palcsó B, Zelkó R. Different types, applications and limits of enabling excipients of pharmaceutical dosage forms. Drug Discov Today Technol 2018;27:21-39.

34. Plöger GF, Hofsäss MA, Dressman JB. Solubility determination of active pharmaceutical ingredients which have been recently added to the list of essential medicines in the context of the biopharmaceutics classification system-biowaiver. J Pharm Sci 2018;107:1478-88.

35. Phulzalke SB, Kate BA, Bagade MY. Solubility Enhancement of telmisartan using mixed hydrotropy approaches. Asian J Biomed Pharm
Sci 2015;5:37-9

36. Pande VV. Exploration of mixed hydrotropy strategy in formulation and development of etodolac injection. J Nanomed Res 2016;3:423.

37. Qi X, Zhang J, Wang W, Cao D. Solubility and stability of indomethacin in arginine-assisted solubilization system. Pharm Dev Technol 2013;18:852-5.

38. Singh N, Allawadi D, Singh S, Arora S. Techniques for bioavailability enhancement of BCS Class II drugs: A review. Int J Pharm Chem Sci 2013;2:38-46.

39. Shelke PS. 2018 Enhancement of solubility of mefenamic acid by hydrotrope based solid dispersion. Int J Sci Res Sci Technol 2010;6:342-9.

40. Sajid AM, Choudhary V. 2012 Solubility enhancement methods with importance of hydrotropy. J Drug Deliv Thera 2013;6:56-62.

41. Solanki SS, Soni LK, Maheshwari RK. Study on mixed solvency concept in formulation development of aqueous injection of poorly water soluble drug. J Pharm (Cairo) 2013;2013:678132.

42. Soni KL, Solanki SS, Maheshwari RK. Development of parenteral formulation of poorly water soluble drugs: The role of novel mixed- solvency concept. Asian J Pharm 2017;11:1-10

43. Ting MJ, Porter WW. Advances in polymer design for enhancing oral drug solubility and delivery. Bioconjug Chem 2014;6:14-22.

44. Takahashi K, Komai M, Kinoshita N, Nakamura E, Hou XL, TakataniNakase T, et al. Application of hydrotropy to transdermal formulations: Hydrotropic solubilization of polyol fatty acid monoesters in water and enhancement effect on skin permeation of 5-FU. J Pharm Pharmacol 2011;63:1008-14.

45. Grishpai K, Divya S, Reddy M, Kumar L, Krishna V. Solubility enhancement of norfloxacin by hydrotropy technique. Int J Pharm Pharm Sci 2014;4:395-7.

46. Rub M, Azum N, Kumar D, Khan F, Asiri A. Clouding phenomenon of amphiphilic drug promazine hydrochloride solutions: Influence of pharamaceutical excipients. J Ind Eng Chem 2015;21:1119-26.

47. Kumar S, Gandhi N. Association model of hydrotropy for the effect of hydrotropes on solubility and mass transfer co-efficient of acetylsalicylic acid. Int J Pharm Pharm Sci 2012;4:600-5.

48. Cui Y. Parallel stacking of caffeine with riboflavin in aqueous solutions: The potential mechanism for hydrotropic solubilization of riboflavin. Int J Pharm 2010;397:36-43.

49. Naqvi AZ, Rub MA, Ud-Din K. Effects of pharmaceutical excipients on cloud points of amphiphilic drugs. J Colloid Interface Sci 2011;361:42-8

50. El-Houssieny BM, El-Dein EZ, El-Messiry HM. Enhancement of solubility of dexibuprofen applying mixed hydrotropic solubilization technique. Drug Discov Ther 2014;8:178-84

51. Subramanian D, Anisimov M. Phase behavior and mesoscale solubilization in aqueous solutions of hydrotropes. Fluid Phase Equilibria 2014;362:170-6.

52. Maheshwari RK, Saxena M, Gahlot M, Chaki R, Kinariwala M, Jagwani Y, et al. Novel application of hydrotropic solubilizing additives in the estimation of aspirin in tablets. Indian J Pharm Sci 2010;72:649-51.

53. Maheshwari R, Manchandani P, Arif P, Mittal D, Man-Chandani P, Indurakhya $\mathrm{A}$, et al. A novel method for quantitative determination of aceclofenac in bulk drug and tablet using ibuprofen sodium as hydrotropic solubilising agents. J Appl Chem Res 2008;5:63-8.

54. Maheshwari R. Analysis of furosemide by application of hydrotropic solubilisation phenomenon. Indian Pharmacist 2005;4:55-8.

55. Maheshwari R, Chaturvedi S, Jain N. Novel application of hydrotropic solubilisation in the analysis of some NSAIDs and their solid dosage forms. Indian J Pharm Sci 2007;69:101-6.

56. Maheshwari RK, Wanare G, Chahar N, Joshi P, Nayak N. Quantitative estimation of naproxen in tablets using ibuprofen sodium as hydrotropic agent. Indian J Pharm Sci 2009;71:335-7.

57. Jayakumar C, Kumar D, Nesakumar D, Gandhi N. Quantitative analysis of theophylline bulk sample using sodium salicylate hydrotrope. Int J Pharm Sci 2010;2:80-1.

58. Jadhav S, Bhatia M, Thamake S, Pishawikar S. Spectrophotometric methods for estimation of atorvastatin calcium form tablet dosage forms. Int J Pharm Tech Res 2010;2:1948-53.

59. El-Khordagui L. Hydrotrope-gelled starch: Study of some physicochemical properties. Int J Pharm 1991;74:25-32.60.

60. Chavda HV, Patel CN, Anand IS. Biopharmaceutics classification system. Syst Rev Pharm 2010;1:62-9. 